\title{
Haemopoietic mechanisms in nasal polyposis and asthma
}

\author{
Judah A Denburg
}

Recent studies of the involvement of the bone marrow in human atopic asthmatic responses to inhaled allergen confirm what we have found in a canine model of Ascaris suum induced bronchial hyperresponsiveness: CD34/45+ haemopoietic progenitors, increased in numbers in the blood and marrow of atopic individuals, can be specifically upregulated following airway allergen challenge eliciting bronchial hyperresponsiveness and the late phase response. These progenitors are also found in nasal polyps ${ }^{1}$ and in asthmatic bronchial mucosa. ${ }^{2}$ An interleukin (IL)-5 responsive subset of progenitors, marking the Eo-B lineage specifically, is upregulated in the marrow within 24 hours of allergen challenge in dual responder asthmatics; using triple colour flow cytometry it can be shown that this subpopulation of progenitors in the marrow is one that bears high affinity receptors for IL-5 (IL-5R $\alpha$ ), existing as a subpopulation of early progenitors bearing CD $34 / 45 .^{3}{ }^{4}$ Thus, a readily mobilisable pool of autocrine (GM-CSF and IL-5 producing ${ }^{5}$ ) Eo-B progenitors at a very early stage of lineage commitment is increased after inhalation of allergen only in those individuals who develop ongoing inflammatory responses. The nature of the signalling between the airway and the bone marrow, which upregulates IL-5R $\alpha$ on $\mathrm{CD} 34+$ progenitors in the bone marrow in vivo, has not been fully clarified. We therefore undertook studies to explore the in vitro regulation of IL-5R expression on haemopoietic progenitors.

\section{IL-5R expression: modulation by retinoic acid}

IL-5 plays a central role in eosinophil and basophil differentiation, exerting its effects through the IL-5 receptor. Although the $\alpha$ chain of the IL-5R is known to exist as either a membrane bound or soluble isoform, little is currently known concerning regulation of IL-5R $\alpha$ gene transcription in the context of commitment of haemopoietic progenitor cells to the eosinophil and basophil lineages.

Recent studies by Tavernier et al have indicated that IL-5 itself can regulate IL- $5 \mathrm{R} \alpha$ expression on cord blood derived mature eosinophils; recent studies in our laboratory indicate that the same holds for bone marrow eosinophil progenitors. Given that all-trans retinoic acid (ATRA) is known to modulate some aspects of haemopoietic differentiation, we examined the effects of ATRA on eosinophil/ basophil differentiation and IL-5R $\alpha$ expression. ATRA selectively inhibited eosinophil/basophil differentiation and was associated with dose dependent inhibition of membrane bound IL-5R $\alpha$ and upregulation of soluble IL- $5 \mathrm{R} \alpha$ transcription (Upham et al, unpublished).

\section{Effects of corticosteroids on eosinophil} progenitors in nasal polyposis and asthma Early studies on the effects of corticosteroids in vitro on eosinophil/basophil differentiation showed a marked dose dependent suppression at concentrations as low as $10^{-9} \mathrm{M} .^{6}$ Studies in the canine model of allergen induced airway inflammation and bronchial hyperresponsiveness have indicated that inhaled corticosteroids suppress the upregulation of myeloid progenitors in the bone marrow ${ }^{7}$ and also the elaboration of a serum haemopoietic activity released within 24 hours of allergen challenge. ${ }^{8}$ In more recent human studies, however, inhaled budesonide failed to suppress the allergen induced upregulation of CD34+IL-5R $\alpha+$ cells in the bone marrow of allergic asthmatic subjects. There was some effect on baseline numbers of CD34+IL-3R+ cells, indicating perhaps that a subset of progenitors was targeted by inhaled corticosteroids in this situation. It is possible that more prolonged treatment with inhaled corticosteroids is necessary to fully suppress the bone marrow contribution to allergic airways inflammation.

In the nasal polyp model, in which CD34+ cells which are capable of myeloid and specifically eosinophil differentiation are present in significant numbers, the effect of intranasal corticosteroids is to increase the CD34+ population further, implying a block in differentiation. ${ }^{1}$

\section{Effects of cysteinyl leukotrienes}

We have found that the cysteinyl leukotrienes $\mathrm{LTD}_{4}$ and $\mathrm{LTE}_{4}$ can both induce eosinophil differentiation of mouse bone marrow progenitors in the presence of either IL-3 or IL-5 in vitro. A leukotriene receptor $\left(\mathrm{LT}_{1}\right)$ antagonist, montelukast, had a moderate suppressive effect on in vitro eosinophil colony formation (unpublished data).

\section{Conclusion}

The studies outlined above indicate that several anti-allergic agents have differential effects in regulating the expression of IL- $5 \mathrm{R} \alpha$ and of eosinophil progenitors in several models of allergic airways disease. The functional consequence in vitro and in vivo of this regulatory effect on IL-5R $\alpha$ is a modulation of eosinophil/ basophil differentiation. Thus, the therapeutic benefit of agents such as corticosteroids, leukotriene antagonists, and retinoids in allergic airways inflammation may be dependent upon the degree to which eosinophil differentiation is modulated. The invaluable help of my co-workers and collaborators (see
references) is much appreciated. This work was funded by the Medical Research Council of Canada. I am grateful for the assistance of Lynne Larocque with the manuscript. 
1 Kim YK, Uno M, Hamilos DL, et al. Immunolocalization of CD34 in nasal polyposis. Effect of topical corticosteroids. Am F Respir Cell Mol Biol 1999;20:388-97.

2 Robinson DS, Damia R, Zeibecoglou K, et al. CD34+ interleukin-5R $\alpha$ messenger RNA+ cells in the bronchial mucosa in asthma: potential airway eosinophil progenitors. Am f Respir Cell Mol Biol 1999;20:9-13.

3 Sehmi R, Howie K, Sutherland DR, et al. Increased levels of CD34+ hemopoietic progenitor cells in atopic subjects. $A m$ f Respir Cell Mol Biol 1996;15:645-54.

4 Sehmi R, Wood LJ, Watson R, et al. Allergen-induced increases in IL- 5 receptor $\alpha$-subunit expression on bone marrow-derived CD34+ cells from asthmatic subjects. A novel marker of progenitor cell commitment toward eosinophilic differentiation. F Clin Invest 1997;100:246675.
5 Gauvreau GM, O'Byrne PM, Mogbel R, et al. Enhanced expression of GM-CSF in differentiating eosinophils of atopic and atopic asthmatic subjects. Am f Respir Cell Mol Biol 1998;19:55-62.

6 Linden M, Svensson C, Andersson M, et al. Circulating eosinophil/basophil progenitors and nasal mucosal cytokines in seasonal allergic rhinitis. Allergy 1999;54:212-9.

7 Woolley MJ, Denburg JA, Ellis R, et al. Allergen-induced changes in bone marrow progenitors and airway responsiveness in dogs and the effect of inhaled budesonide on these parameters. Am f Respir Cell Mol Biol 1994;11:600-6. 8 Inman MD, Denburg JA, Ellis R, et al. Allergen-induced increase in bone marrow progenitors in airway hyperreAm f Respir Cell Mol Biol 1996;15:305-11. 